\title{
ACUERDO COMERCIAL CON LA UNIÓN EUROPEA: OPORTUNIDAD DE CRECIMIENTO DEL SECTOR CONFECCIONES DESDE LA RESPONSABILIDAD SOCIAL EMPRESARIAL
}

\section{COMMERCIAL AGREEMENT WITH THE EUROPEAN UNION: OPPORTUNITY FOR THE GROWTH OF THE CLOTHING SECTOR'S FROM SOCIAL RESPONSIBILITY BUSINESS}

\section{Diana Patricia Gutiérrez Mejía' Laura Lucía Colmenares Botía ${ }^{2}$}

\section{RESUMEN}

Este trabajo resalta la importancia de las exportaciones del sector confecciones dentro del crecimiento de este y la relevancia de las relaciones comerciales entre Colombia y la Unión Europea frente a su aporte al desarrollo económico, esto a través del Sistema de Preferencias Generalizadas (SPG), el cual incluye temas sociales, laborales, de medio ambiente y buena gobernanza económica, entre otros (Mincit, 2012). Por tanto, los programas de Responsabilidad Social Empresarial (RSE) de las empresas del sector se han convertido en una de las herramientas determinantes a la hora de aprovechar las oportunidades comerciales dentro del acuerdo, lo que genera que algunas empresas tomen fuerza en este mercado.

Palabras clave: Responsabilidad Social Empresarial, mercado común, bienestar social, acuerdo comercial (EU), cooperación internacional.

\section{ABSTRACT}

This project highlights the importance of the clothing sector's exports, its growing and the relevance of the commercial relationship between Colombia and the European Union for its contribu-

1 Docente investigadora de la Universidad Piloto de Colombia. Correo electrónico: diana-gutierrez4@unipiloto.edu.co Orcid: https://orcid.org/0000-0003-1673-508X

2 Docente investigadora de la Universidad Piloto de Colombia. Correo electrónico: laura-colmenares@unipiloto.edu.co Orcid: https://orcid.org/0000-0002-5977-4372

Código JEL: F14, M14, C13.

Fecha de recepción: 17/08/2018

Fecha de aprobación: 4/09/2018

DOI: https://doi.org/10.18601/16577175.n22.06 
tion to economic development, by using the Generalized System of Preferences (GSP) wich includes, social, labor, environment, governance and economic topics among many others (Mincomercio, industria y turismo, 2012). Therefore the sector's companies' programs of Corporative Social Responsibility (CSR) have become one of the most important tools to take advantage of the comercial opportunities within the agreement, it have brought the strengthening of some companies in the market.

Keywords: corporate social responsibility, common market, social wellness, trade agreement (EU), international cooperation.

\section{INTRODUCCIÓN}

La desaceleración del consumo, el contrabando y el aumento de importaciones configuran un panorama actual no muy alentador para la industria de confecciones $^{3}$ colombiana, no obstante, el acuerdo comercial con la Comunidad Europea se ha convertido en una gran oportunidad para el crecimiento del sector y del país, ya que ha permitido el aumento de las exportaciones. Frente a esto, se han evidenciado casos exitosos de empresas del sector, que están posicionadas en nichos de mercado específicos, las cuales han utilizado herramientas de valor agregado y alto reconocimiento internacional, lo que les permite sobrevivir en el entorno doméstico y conquistar de igual forma mercados internacionales.
El acuerdo comercial Colombia-Unión Europea estimula el desarrollo sostenible por medio del Sistema de Preferencias Generalizadas (SPG) (Comisión Europea, 2004), esto por medio del comercio como motor económico. A partir de lo anterior, los empresarios del sector buscaron una mayor competitividad y sostenibilidad, comenzando con un constante compromiso con la responsabilidad social y la incorporación de programas para cuidar y preservar el medio ambiente, la salud y la seguridad de los trabajadores, con el fin de evitar situaciones de riesgo, las cuales a largo plazo pueden ir en detrimento de sus organizaciones (Comisión Europea, 2018, p. 1).

Dichos elementos llevaron a definir este trabajo, el cual busca establecer cómo las exportaciones del sector de confecciones en Colombia son importantes para el crecimiento del sector y cómo el diseño de una alternativa de internacionalización para las empresas del sector de la mano de la Responsabilidad Social Empresarial permitirían aprovechar las oportunidades comerciales que ofrece el acuerdo comercial con la Unión Europea. Lo anterior refleja, a su vez, un aporte al desarrollo sostenible dadas las normas establecidas en el Sistema de Preferencias Generalizadas (SPG) de la EU.

En principio se tomará un modelo econométrico que permitirá establecer la importancia de las exportaciones en el crecimiento del sector, luego se 
realizará un análisis de la importancia y la fuerza que ha tomado la Responsabilidad Social Empresarial en el sector $\mathrm{y}$, por último, se efectuará un primer acercamiento a un modelo de internacionalización que contenga herramientas de la RSE con formas de organización que maximicen la comercialización y sostenibilidad del sector. Por tal motivo se busca responder la siguiente pregunta: ¿cómo estructurar una estrategia de internacionalización a partir de variables como la Responsabilidad Social Empresarial, que permitan fortalecer las exportaciones para así aprovechar las oportunidades del acuerdo comercial con la Unión Europea en pro del crecimiento del sector de confecciones en Colombia?

\section{MATERIALES Y MÉTODOS}

A continuación, se busca sustentar la importancia de la innovación y otras variables en las estrategias de internacionalización del sector de confecciones en Colombia, con miras al desarrollo de trasformaciones que beneficien a la población involucrada. Bajo este contexto se resalta la importancia de las exportaciones desde las teorías de crecimiento, las cuales se sustentan en que estas permiten evidenciar un aumento del PIB ${ }^{4}$. Frente a esto, Thirlwall (2011), a través de la teoría de restricción de balanza de $\operatorname{pagos}^{5}$, afirma que las exportaciones al ser la demanda que no depende del ingreso interno pueden servir de multiplicador del crecimiento, esto lo demuestra a través de la tasa de crecimiento en el largo plazo, ya que dependerá de la diferencia entre la tasa de los ingresos (exportaciones) y egresos (importaciones) generada por el comercio internacional. Esta condición se llama la Ley de Thirlwall (Márquez, 2009). Su modelo básico o regla simple se formaliza en la siguiente ecuación:

$y_{b r s c} \frac{(\varepsilon z+(\pi+\gamma) c)}{\eta}$

\section{Donde}

y: tasa de crecimiento del Producto Interno Bruto (PIB) interno;

$\varepsilon$ : elasticidad ingreso de la demanda de las exportaciones;

$z$ : tasa de crecimiento del PIB externo;

$\pi$ : elasticidad negativa precio de la demanda por exportaciones;

$\gamma$ : elasticidad negativa precio de la demanda por importaciones;

c: tasa de cambio real;

$\eta$ : elasticidad ingreso de la demanda de las importaciones (Clavijo y Ros, 2015).

Thirlwall (2011) concluye que para la mayoría de los países que deseen crecer más rápido, primero deben elevar la restricción de la balanza de pagos y elevar también la tasa de crecimiento de la capacidad productiva, para lo cual deben hacer "las exportaciones más atractivas y reducir la elasticidad- 
ingreso de la demanda de importaciones, la demanda puede expandirse sin producir dificultades en la balanza de pagos; y, dentro de ciertos límites, la demanda puede generar su propia oferta fomentando la inversión, absorbiendo el subempleo y aumentando el crecimiento de la productividad" (Thirlwall, 2011, p. 429), entre otros. Este autor afirma que su modelo y la evidencia empírica brindan un fuerte apoyo a los defensores del crecimiento liderado por las exportaciones. Finalmente, que:

Para los países con una tasa baja de crecimiento de las exportaciones, combinada con una elasticidad ingreso relativamente alta de la demanda de importaciones, el mensaje es claro: los bienes producidos por el país son relativamente poco atractivos tanto en el país como en el extranjero [...] El argumento probablemente tenga una relevancia aún mayor para los países en desarrollo. (Thirlwall, 2011, p. 438)

Márquez, por su parte, en el 2008, demuestra que la Ley de Thirlwall (2011) es más adecuada para explicar la relación entre el comercio exterior y el crecimiento de una economía como la colombiana (p. 23), de tal modo convierte este modelo de crecimiento restringido por balanza de pagos como un buen estimador (p. 53) que, a su vez, retroalimenta el modelo anterior.

Bajo este panorama es necesario replantear el alcance de las empresas al incursionar al mercado mundial en la utilización de su máxima capacidad operativa y el aumento de su eficiencia en la producción, con el fin de "asegurar su supervivencia en un mundo altamente globalizado" (De la Vega, 2017, p. 2) y, de esta manera, poder "operar de manera más eficiente y efectiva y mejorar su desempeño, actuando en los mercados antes que sus competidores" (De la Vega, 2017, p. 6). Todo, sin dejar de lado la RSE, la cual se ha convertido en una herramienta fundamental a la hora de buscar el desarrollo sostenible y de tener legitimidad en el sistema comercial trasfronterizo.

La preocupación por generar "desarrollo sustentable" de forma oficial a nivel mundial se da por primera vez en la Declaración de Estocolmo (1972, principio 2), pero con los años se determinó que se debía ir más allá de los recursos naturales, se debe preservar la cultura, las necesidades sociales, la economía del ser humano. Por esta razón, en 1987, se empezó a hablar de" desarrollo sostenible", ya que por medio de este se busca satisfacer las necesidades, como alimentación, cultura, sociales, la economía del ser humano y de forma que perdure en el tiempo, por lo que en la Declaración de Johannesburgo (2002) se definió al desarrollo sostenible como el "Proceso mediante el cual se satisfacen las necesidades económicas, sociales, de diversidad cultural y de un medio ambiente sano de la actual generación, sin poner en riesgo la satisfacción de las mismas a las generaciones futuras". Lo que le permite al ser humano "vivir bien", que es "el equilibrio material y espiritual del individuo (saber vivir) y la relación armoniosa del mismo con todas las formas de existencia (convivir)" (Huanacuni, 2010, p. 15), convirtiéndose en 
un elemento fundamental de la realidad mundial actual.

Este contexto se materializa, en el 2000, al instaurarse los Ocho Objetivos de Desarrollo del Milenio (ODM) por parte de Naciones Unidas (ONU) para abordar temas como la pobreza, el hambre, la educación, el desarrollo, el pleno empleo y el crecimiento económico. En 2012, se establecieron los Diecisiete Objetivos de Desarrollo Sostenible (ODS) en la Conferencia de las Naciones Unidas sobre el Desarrollo Sostenible en Río de Janeiro, con el propósito de dar cumplimiento a los ODM en virtud de la asignación de metas más específicas que reunieran la acción colectiva de los Estados para solventar las problemáticas globales que estos abarcan, los cuales se articularon con otro acuerdo histórico celebrado en el 2015, el Acuerdo de París, aprobado en la Conferencia sobre el Cambio Climático (COP21), junto con el Marco de Sendai para la Reducción del Riesgo de Desastres, firmado en Japón en marzo del 2015 (PNUD, 2018).

Por su parte, Amartya Sen, en su trabajo "El desarrollo como libertad" (2000), plantea que "el desarrollo puede ser considerado como un proceso de expansión de las libertades reales que disfruta la gente" (p. 15). Con lo que busca ampliar el enfoque sobre el desarrollo, puesto que, según el autor, este va más allá del crecimiento del producto nacional bruto (PNB), el aumento de los ingresos per cápita, la industrialización, el avance tecnológico o la modernización social. No obstante, no desconoce que estas variables son importantes para ampliar las libertades de las que disfrutan los miembros de la sociedad, sin embargo, estima la necesidad de observar otros factores, como los planes sociales y económicos, dentro de los cuales se encuentran aquellos orientados a la educación y al cuidado de la salud; los derechos civiles y políticos y demás variables que contribuyen sustancialmente a la expansión de la libertad humana. Además, afirma que el desarrollo requiere de la eliminación de importantes fuentes de la ausencia de libertad como son: "pobreza y tiranía, oportunidades económicas escasas y privaciones sociales sistemáticas, falta de servicios públicos, intolerancia y sobre actuación de estados represivos" (p. 15).

Otro concepto importante que se aborda dentro de este trabajo es el de Responsabilidad Social Empresarial (RSE) el cual empieza a aparecer a finales del siglo XIX, en la búsqueda por articular la interacción empresarial y social en pro del desarrollo social y económico. Algunos la definen como "una filosofía corporativa adoptada por la alta dirección de la empresa para actuar en beneficio de sus propios trabajadores, sus familias y el entorno social en las zonas de influencia de las empresas" (CEDIS, 2009). Por su parte, Correa (2007) manifiesta que la RSE viene dada por fases: la primera se dio a finales del siglo XIX, en la que era entendida como la manera en la que las empresas solucionaban las problemáticas que se presentaban en su entorno. La segunda, se presenta a mitad del siglo XX de forma "filantrópica" asociada a actividades que coadyuvaban al bienestar del individuo. Finalmente, la tercera, 
se presenta en la última mitad del siglo XX y se reguló mediante leyes para que el gobierno y la empresa trabajaran de forma conjunta por el bienestar social de todos los individuos.

Desde la perspectiva económica y empresarial, Friedman (1970), citado por Ekornes (2013), afirma que la maximización de las utilidades de los accionistas $^{6}$ dependerá de algunas restricciones a las que se enfrentan las empresas como las normas sociales, las costumbres y las leyes, entre otras. Por lo que manifiesta que "uno debe cumplir con la ley incluso si uno no está de acuerdo con ella, ya que aplica a la maximización del beneficio y cuando se trata de externalidades negativas la empresa debe obtener su beneficio, pero no a expensas de otras personas o empresas" (Ekornes, 2013).

Por su parte, Hosseini y Brenner (1992) consideran que las empresas tienen una visión más generalizada en relación con el bienestar, puesto que "el interés de las empresas se centra en las relaciones intergrupales en cuanto a las actitudes y valores que surjan de la interacción entre empresa e individuos concentrándose en los reclamos del quehacer de la empresa en el contexto" (p. 102).

En esta misma línea, Garriga y Melé (2004) enfatizan que las empresas asumen criterios de responsabilidad social de acuerdo con la forma en la que se desempeñan en el mercado. Por lo que la RSE puede categorizarse de acuerdo con el contexto en el que se desenvuelven las empresas y puede requerirse de intervención privada o pública para mitigar los impactos o externalidades negativas en el contexto social:

1. Respecto a la ganancia, en la que se trabaja en virtud de un rendimiento o beneficio en el corto plazo.

2. La política, en la que las normas decretan lo necesario e importante para que las empresas lleven a cabo su actividad comercial sin dejar de lado el bienestar social.

3. El negocio, por medio de las agremiaciones, las cuales buscan identificar las necesidades y beneficios del colectivo y de su entorno.

4. La ética, en la que realmente se observa el verdadero hacer de la empresa en el entorno y lo que la RSE conlleva en su ejercicio empresarial.

Por otro lado, es importante abordar el tema de los tratados comerciales, ya que son un instrumento muy importante a la hora de querer aumentar las exportaciones, debido a que como resultado de la política de internacionalización de la economía colombiana a la fecha se posee una red de quince acuerdos comerciales vigentes, dos suscritos y tres en negociación (tabla 1) (Mincit, 2018).

6 Ekornes (2013) hace uso del término de accionistas - stockholder - a todas aquellas figuras que intervienen el funcionamiento de una empresa desde el punto de vista monetario, llámense ejecutivos, accionistas, dueños, entre otros. 
Tabla 1.

Acuerdos comerciales Colombia

\begin{tabular}{|l|c|c|c|}
\hline \multicolumn{1}{|c|}{ Acuerdos } & Vigente & Suscrito & En negociación \\
\hline TLC Colombia - México & $\mathrm{X}$ & & \\
\hline El Salvador, Guatemala y Honduras & $\mathrm{X}$ & & \\
\hline CAN & $\mathrm{X}$ & & \\
\hline CARICOM & $\mathrm{X}$ & & \\
\hline MERCOSUR & $\mathrm{X}$ & & \\
\hline Chile & $\mathrm{X}$ & & \\
\hline EFTA & $\mathrm{X}$ & & \\
\hline Canadá & $\mathrm{X}$ & & \\
\hline Estados unidos & $\mathrm{X}$ & & \\
\hline Acuerdo de alcance parcial con Venezuela & $\mathrm{X}$ & & \\
\hline Cuba & $\mathrm{X}$ & & \\
\hline Unión Europea & $\mathrm{X}$ & & \\
\hline Corea & $\mathrm{X}$ & & \\
\hline Costa Rica & $\mathrm{X}$ & & \\
\hline Israel & & $\mathrm{X}$ & \\
\hline Panamá & & $\mathrm{X}$ & \\
\hline Turquía & & & $\mathrm{X}$ \\
\hline Japón & & & $\mathrm{X}$ \\
\hline TISA & & & \\
\hline
\end{tabular}

Fuente: elaboración propia a partir de la información de Tratados de Libre Comercio (Mincit, 2018).

Dentro de los vigentes, se resalta el acuerdo con la Unión Europea expuesto en el apartado de resultados.

\section{METODOLOGÍA}

Este trabajo se deriva del desarrollo de las primeras fases de una investigación con mayor alcance en la cual se cuenta con el apoyo de la Federación Nacional de Comerciantes (Fenalco) $)^{7}$. Tanto la investigación como este avance se realiza bajo un enfoque metodológico mixto, su alcance de profundidad es explicativo ${ }^{8}$, ya manipula variables que influyen en el crecimiento del sector confecciones, con miras a proponer una estrategia de internacionalización que tenga en cuenta la RSE como un instrumento de desarrollo sostenible y que le permita aprovechar las oportunidades que ofrece el acuerdo comercial con la Unión Europea. Su objeto de estudio son las empresas del mencionado sector en Colombia, por tanto, en esta primera fase se acudirá

7 Es una entidad gremial, de carácter permanente, sin ánimo de lucro, encargada de fomentar el desarrollo del comercio y de orientar, representar y proteger sus intereses, dentro de un criterio de bienestar y progreso del país, donde prima la solidaridad gremial, la eficiencia y la modernización de los empresarios colombianos.

8 Según Sampieri, es explicativo porque además de explicar el porqué de las cosas propone una solución. 
a información secundaria de bases de datos fiables del sector público y privado. Se debe resaltar que el proyecto se desarrollará en tres fases nombradas, a continuación, y actualmente se encuentra en la tercera.

Fase I, proceso de sensibilización: la investigación comenzó con una reunión con los directivos que se interesaron por esta, pues consideran que existe una problemática en el sector que necesita ser tratada de forma urgente.

Fase II, revisión documental: esta se realiza de forma rigurosa sobre la importancia de las exportaciones en el crecimiento del sector y el país, el aprovechamiento de las oportunidades que brinda el acuerdo comercial con la Unión Europea y de qué manera el sector ha abordado el tema de RSE como herramienta para el fomento del desarrollo sostenible. Esto a través de artículos científicos e investigaciones de alta calidad y consulta en bases de datos académicas y científicas, además, se contó con el acompañamiento de Fenalco.

Fase III: se determina un primer modelo econométrico para determinar la importancia de las exportaciones del sector en su crecimiento y del país, el cual se modela en el programa Stata y a partir de esto y los resultados obtenidos en la Fase II se propondrá un nuevo modelo, dentro del cual se tendrá en cuenta la RSE, además, se presentarán las primeras conclusiones en torno a este tema y un breve análisis prospectivo del sector como soporte para la propuesta de dicho modelo.
El presente trabajo va hasta esta fase, pero la investigación macro continúa con la determinación del instrumento de recolección de información, ya sea entrevista estructurada y encuestas, el cual se busca validar por expertos en el tema. Una vez recolectada la información, se tabula con el fin de correrla en el programa Stata y determinar la significancia de las variables determinadas.

Fase IV: a partir de los resultados obtenidos antes, se plantea la estrategia de internacionalización que vincula al Estado y la empresa privada para finalmente plantear las conclusiones y recomendaciones que den cumplimiento al objetivo general.

\section{RESULTADOS Y DISCUSIÓN}

\section{a. Contexto del sector en torno al acuerdo comercial con la Unión Europea}

Anterior a la contextualización del sector es necesario citar las generalidades del acuerdo comercial con la Unión Europa, el cual fue firmado en la ciudad de Bruselas, Bélgica, el 26 de junio del 2012, y aprobado por el Parlamento Europeo el 11 de diciembre del mismo año, y posteriormente notificó la culminación de sus trámites internos para la aplicación provisional del acuerdo el 27 de febrero del 2013. El presidente de la República de Colombia mediante el Decreto 1513 del 18 de julio del 2013 dio aplicación provisional al acuerdo comercial. El 5 de noviembre del 2014 el Gobierno nacional expidió el Decreto 2247 "a través del cual se señala que el país continuará aplicando sin solución 
de continuidad, en los términos señalados en el Decreto 1513 de 2013, el Acuerdo Comercial suscrito con la Unión Europea y sus Estados miembros", luego de haberse cumplido con todos los requisitos internos previstos en la ley para la aprobación del mismo (Mincit, 2018).

Dicho acuerdo establece la reducción de barreras arancelarias y otros temas técnicos que facilitan el comercio, como son medidas sanitarias y fitosanitarias, inversiones, asuntos laborales, disposiciones para el medio ambiente, propiedad intelectual, entre otros. Así mismo, el acuerdo busca fortalecer lo comercial y contribuir a "la consolidación de la democracia, el respeto a los derechos humanos, la lucha contra la pobreza, el aumento del empleo en condiciones decentes, el compromiso con el medio ambiente y el desarrollo sostenible" (Delegación de la Unión Europea en Colombia y Mincit, 2012, p. 19).

Lo anterior, lo realiza la Unión Europea mediante el Sistema de Preferencias Generalizadas (SPG), el cual ofrece aranceles más bajos o acceso en franquicia de derechos al mercado de la EU a las importaciones procedentes de 178 países y territorios en desarrollo. Además, ofrece beneficios especiales a 49 países menos desarrollados y a países que cumplen determinadas normas básicas en el ámbito laboral y en el del medio ambiente, esto sin exigir contrapartidas a los países beneficiarios (Comisión Europea, 2004, p. 2). De igual manera,
Colombia se ve beneficiada del régimen especial de estímulo del desarrollo sostenible conocido como SPG+, el cual otorga preferencias adicionales a un grupo de dieciséis países con el objetivo de apoyar a los países en vías de desarrollo vulnerables en su ratificación y aplicación de los convenios internacionales clave en materias de derechos humanos, sociales y laborales, así como de medio ambiente y buena gobernanza económica (Mincit, 2012).

Este sistema se basa en la Declaración de Johannesburgo sobre desarrollo sostenible, del 2002, por lo que se promueve un enfoque global que tenga en cuenta componentes sociales, medioambientales y económicos que lleven a la responsabilidad colectiva para así promover y fortalecer en los planos local, nacional, regional y mundial el desarrollo económico, social y la protección ambiental, pilares interdependientes y sinérgicos del desarrollo sostenible. Lo que se evidencia en un acuerdo comercial comprometido con estos grandes retos en un mundo globalizado, buscando avances y reforzando sus tres pilares básicos ${ }^{9}$ (Mincit, 2012).

A partir de este contexto se resalta la realidad del sector de confecciones en el marco de mencionado acuerdo, el cual le ha permitido configurar un escenario de oportunidades dentro del continente europeo. Esto se refleja en el pasado informe de Procolombia (2017) en el que se estima que del total de exportaciones que se realizaron a la Unión 
Europea en el 2016, el 3,3\% correspondió al sector de confecciones, estas exportaciones tuvieron como destino veinte mercados distintos, cuyos focos comerciales exigen diversificación en los productos, calidad y competencia a nivel de precios. A continuación, se exponen los principales mercados de la Unión Europea para el sector de confecciones o moda junto con los productos que estos mercados demandan (figuras 1 y 2 ).

Figura 1.

Principales mercados de la EU destino de los productos de la cadena sistema moda en el 2016

Italia:

- US\$28,1 millones

- Participación en la UE: $37,1 \%$

Espaగึa:

- US\$16 millones

- Participación en la UE: $21,2 \%$

Paises Bajos:

- US\$12,9 millones

- Participación en la UE: $17,1 \%$

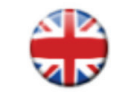

Reino Unido:

- US\$6,2 millones

- Participación en la UE: $8,1 \%$

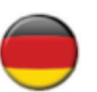

\section{Alemania}

- US\$4,7 millones

- Participación en la UE: $6,2 \%$

Fuente: (Procolombia, 2017)

Figura 2.

Mercados de oportunidad europeos para la cadena de sistema moda

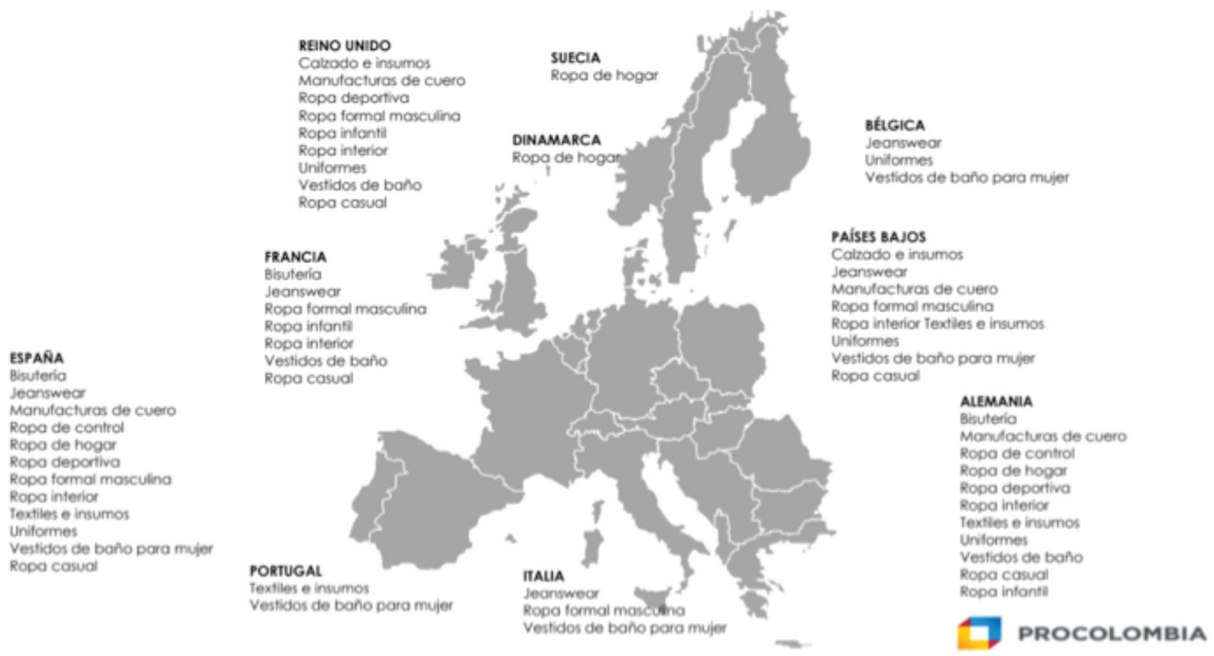

Productos: Cueros en bruto y preparados, ropa formal masculina, jeanswear, textiles e insumos, vestidos de baño, manufacturas de cuero, bisuteria, vestidos de baño y ropa de control.

Productos: Textiles e insumos, pieles de babilla, Cueros en bruto y preparados, jeanswear, ropa de control, manufacturas de cuero y ropa interior.

Productos: Jeanswear, ropa formal masculina, peleteria, ropa casual, ropa interior y vestidos de baño.

Productos: Manufacturas de cuero, ropa infantil, jeanswear, ropa formal masculina, textiles e insumos, ropa interior, ropa de control y vestidos de baño

Productos: Ropa de hogar, jeanswear, manufacturas de cuero, vestidos de baño, cueros en bruto y preparados y bisutería 
De igual forma se destaca que el con productos en las categorías de sector de confecciones fue el que jeanswear, ropa formal masculina, más exportaciones presentó, con un ropa de hogar, vestidos de baño, fajas total exportado representado en 32,5 y ropa de control, entre otros. Dicha millones de dólares en el 2016, en la información se puede observar en la actualidad sigue siendo el principal figura 3 expuesta a continuación (Prosector de exportación de la cadena, colombia, 2017).

Figura 3.

Principales productos exportadores de la cadena de sistema moda a la EU 2016; (USD) Free on Board (libre a bordo, puerto de carga convenido) (FOB)

\begin{tabular}{|l|r|c}
\hline \multicolumn{1}{|c|}{ Producto } & US\$ FOB 2016 & Part. \% 2016 \\
\hline Cueros en bruto y preparados & 28.667 .341 & $37,90 \%$ \\
\hline Otros textiles y confecciones & 8.623 .387 & $11,40 \%$ \\
\hline Jeans & 7.854 .055 & $10,4 \%$ \\
\hline Ropa formal masculina & 5.056 .144 & $6,7 \%$ \\
\hline Textiles & 4.459 .515 & $5,9 \%$ \\
\hline Manufacturas de cuero & 3.292 .270 & $4,4 \%$ \\
\hline Ropa de hogar & 2.319 .959 & $3,1 \%$ \\
\hline Vestidos de baño femeninos & 2.094 .026 & $2,8 \%$ \\
\hline Pieles de babilla & 2.068 .688 & $2,7 \%$ \\
\hline Bisuteria & 1.704 .817 & $2,3 \%$ \\
\hline Fajas y ropa de control & 1.643 .093 & $2,2 \%$ \\
\hline T-shirts camisetas & 1.491 .687 & $2,0 \%$ \\
\hline Peleteria (cuero manufacturas de cuero) & 1.214 .271 & $1,6 \%$ \\
\hline Ropa interior femenina & 1.073 .936 & $1,4 \%$ \\
\hline Calzado & 956.378 & $1,3 \%$ \\
\hline Suéteres & 832.925 & $1,1 \%$ \\
\hline Ropa de bebe-infantil & 809.808 & $1,1 \%$ \\
\hline Orfebrería & 758.471 & $1,0 \%$ \\
\hline Ropa interior masculina & 460.075 & $0,6 \%$ \\
\hline Calcetería & 105.018 & $0,1 \%$ \\
\hline Vestidos de baño masculinos & 69.843 & $0,1 \%$ \\
\hline Joyería & 37.366 & $0,0 \%$ \\
\hline Otros & 18.183 & $0,0 \%$ \\
\hline Iotal general & $\mathbf{7 5 . 6 1 1 . 2 5 6}$ & $100 \%$ \\
\hline
\end{tabular}

Fuente: (Procolombia, 2017)

Finalmente, se puede observar cómo ha ido fluctuando el comercio internacional de este sector, desde un énfasis puntual a las importaciones hasta las exportaciones y la balanza comercial (figura 4). 
Figura 4.

Comercio internacional sector confecciones Colombia

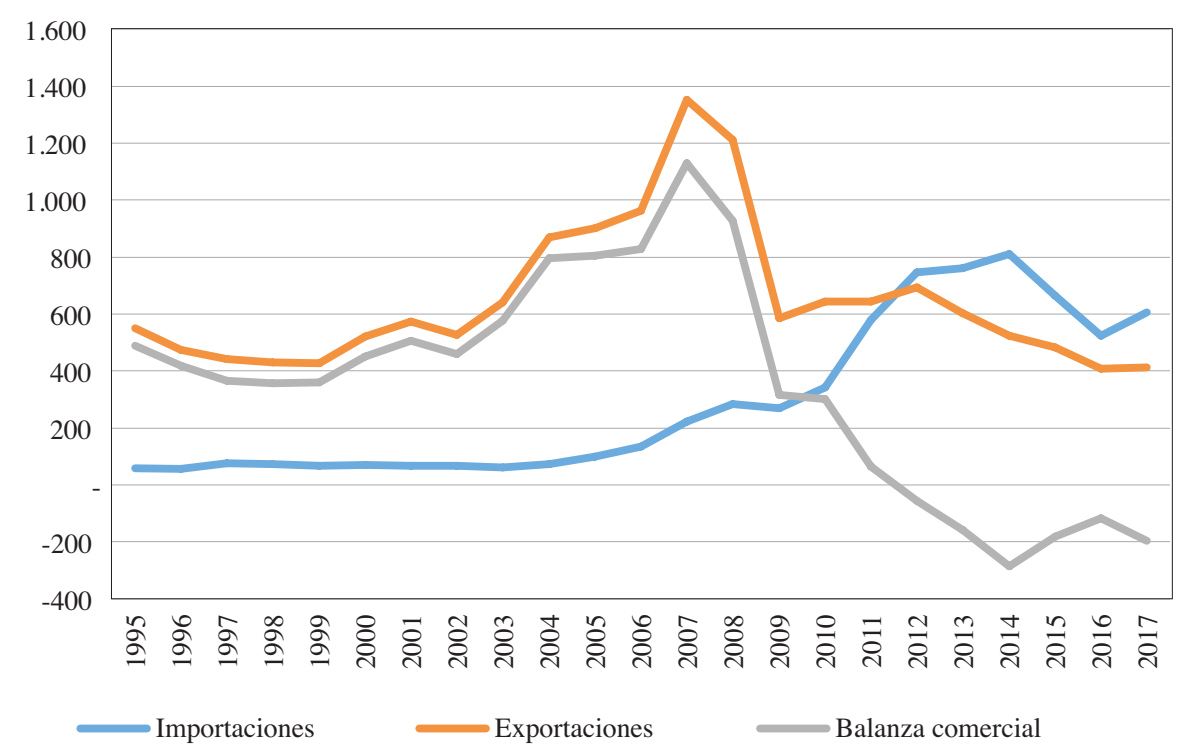

Fuente: elaboración propia a partir de datos del DANE, 2018.

\section{b. El sector confecciones en Colombia y la RSE}

En el sector de confecciones, al igual que muchos otros sectores económicos, las empresas se encuentran motivadas y a la misma vez desafiadas a fomentar un compromiso con la creación de estrategias socialmente responsables, las cuales se materializan en herramientas, programas e iniciativas que permiten crear nuevos empleos, contribuyen a la protección medioambiental, generan condiciones laborales adecuadas para los trabajadores, entre otras. Esto con miras a crear una buena reputación en el entorno comercial y, a su vez, proporcionar un buen clima laboral que a futuro se materializa en ganancias para las empresas del sector.

No obstante, hay muchos factores en la realidad del sector que dificultan la consecución plena de las metas en materia de RSE propuestas por las empresas. Problemáticas como los costos en la mano de obra, la informalidad laboral, la falta de capacitación y formación laboral y dificultades con la normativa plantean un panorama no muy alentador en este aspecto, reflejo de esto son las cifras expuestas por Echavarría (2015):

El sector textil genera 466.000 empleos formales, pero estimaciones de expertos indican que puede haber otro porcentaje de trabajadores informales vinculados al negocio. En otras palabras, casi 1 millón de personas trabaja directamente en esta industria, lo que equivale a que unos 4 millones de colombianos dependen del buen funcionamiento y desarrollo de esta actividad. (p. 82)

Estas cifras, contrastadas con las cifras del ministro de Trabajo, permiten obser- 
var que aproximadamente unas 700000 personas trabajan en la informalidad en el sector textil. Sin duda erradicar este aspecto es todo un reto para la RSE, en virtud de que su objeto consiste en propender condiciones laborales formales que garanticen el pleno cumplimiento de los derechos de los trabajadores.

Adicionalmente, cabe analizar en términos salariales si los pagos son acordes a la realidad del sector y al tiempo desempeñado durante las jornadas laborales, lo que lleva a nuevos cuestionamientos frente a la posibilidad de materializar los objetivos expuestos en un inicio. Por tal motivo, es necesario contar con auditorias, procesos de retroalimentación y procesos de verificación laboral que evidencien la realidad laboral del sector para que se tracen compromisos plausibles que permitan fortalecer aspectos débiles y potencializar virtudes y de esta manera crear escenarios laborales satisfactorios y coherentes con los parámetros dados por la RSE.

De igual manera, con la creación de modelos como el propuesto, se pretende responder a este tipo de coyunturas desde el involucramiento con el sector para obtener datos reales que permitan arrojar resultados y estrategias de mejora acordes con las necesidades de los trabajadores y de las empresas.

A continuación, se presentan los principios de RSE implantados en la industria textil y de confecciones en Colombia (tabla 2).

Tabla 2

Principios de RSE industria textil y de confecciones en Colombia

\begin{tabular}{|c|c|}
\hline $\begin{array}{l}\text { Derechos } \\
\text { humanos }\end{array}$ & $\begin{array}{l}\text { El } 38 \% \text { de las empresas exige certificaciones a sus proveedores en los que } \\
\text { se comprometen a prohibir todo tipo de discriminación a empleados, trabajo } \\
\text { forzoso e infantil, no implementar procesos que afecten el medio ambiente y } \\
\text { no vulnerar los derechos humanos. }\end{array}$ \\
\hline \multirow{3}{*}{$\begin{array}{l}\text { Normatividad } \\
\text { laboral }\end{array}$} & $\begin{array}{l}\text { El } 85 \% \text { invierten recursos extrasalariales para contribuir a la educación, vivienda } \\
\text { y recreación de sus empleados, entre otros. }\end{array}$ \\
\hline & $\begin{array}{l}\text { Se promulga la contratación de empresas formales en las que se genere el cum- } \\
\text { plimiento de las leyes laborales vigentes. }\end{array}$ \\
\hline & $\begin{array}{l}\text { Alta contratación de personal en situación de desventaja como madres cabeza } \\
\text { de familia, reinsertadas, desplazadas y reclusas, entre otros. }\end{array}$ \\
\hline \multirow{2}{*}{$\begin{array}{l}\text { Medio } \\
\text { ambiental }\end{array}$} & $\begin{array}{l}\text { En pro del desarrollo sostenible han nacido empresas como My Green Life, } \\
\text { quienes fabrican etiquetas a partir de una planta que crece en la maleza de las } \\
\text { represas, confeccionan muñecos de retazos de telas, usan colorantes naturales } \\
\text { que no contaminan, realizan estampados libres de sustancias químicas nocivas } \\
\text { para la salud y tercerizan los procesos de confección con fundaciones. }\end{array}$ \\
\hline & $\begin{array}{l}\text { Compañías como ENKA y Fabricato crean fibras utilizando poliéster reciclado, } \\
\text { además, las empresas del sector presentan en sus etiquetas la totalidad de las } \\
\text { materias primas utilizadas. Se han creado programas de reciclaje y de disminu- } \\
\text { ción de consumo de agua y energía dentro del proceso productivo. }\end{array}$ \\
\hline $\begin{array}{l}\text { RSE con la } \\
\text { comunidad }\end{array}$ & $\begin{array}{l}\text { El } 58 \% \text { de las empresas en el } 2012 \text { destinaron recursos a la inversión social y a } \\
\text { donaciones a la comunidad, siendo estos de aproximadamente el 0,14\% de los } \\
\text { ingresos operacionales ( } 97,63 \text { millones por empresa). }\end{array}$ \\
\hline
\end{tabular}

Fuente: Morales (2016) a partir de información de la Organización Pacto Mundial. 
Según Morales (2016), en Colombia, el sector textil ha sido pionero de RSE, desde las épocas de antaño, aun así debe fortalecerse esta área dada su importancia dentro del desarrollo sostenible y las oportunidades que genera para el sector dentro del acuerdo comercial con la EU, dados los lineamientos del régimen especial de estímulo del desarrollo sostenible (SGP+).

Importancia de las exportaciones dentro del crecimiento del sector y el país

Con el fin de determinar el efecto de las exportaciones del sector en su crecimiento, se propone en principio un modelo econométrico derivado de la

Ley de Thirlwall:

$\ln y=\beta_{0}+\beta_{1} \ln x+\beta_{2} \ln c+\beta_{3} \ln \eta+\xi$

\section{Donde}

$y$ : tasa de crecimiento del PIB interno sector confecciones;

$\mathrm{x}$ : exportaciones sector confecciones;

c: índice de la tasa de cambio real;

$\eta$ : elasticidad ingreso de la demanda de las importaciones sector confecciones; $\xi$ : error estocástico.

Al modelar los datos en el programa Stata 11 se obtienen los siguientes resultados (ver tabla 3 ).

Tabla 3

Resultados del modelo

\begin{tabular}{|l|c|c|c|c|c|c|}
\hline$y$ & Coef. & Std. Err. & $\mathrm{t}$ & $\mathrm{P}>\mathrm{t}$ & [95\% Conf. & Interval] \\
\hline$x$ & 6,209745 & 2,896258 & 2,14 & 0,058 & $-0,2435213$ & 12,66301 \\
\hline$c$ & $-0,0492657$ & 0,0393515 & $-1,25$ & 0,239 & $-0,1369462$ & 0,0384149 \\
\hline$\eta$ & 8,336598 & 19,45701 & 0,43 & 0,677 & $-35,01631$ & 51,68951 \\
\hline- cons & 2,201041 & 4,370307 & 0,5 & 0,625 & $-7,53661$ & 11,93869 \\
\hline
\end{tabular}

Fuente: salida de Stata 11, a partir de datos tomados del DANE y el Banco de la Republica de Colombia.

El modelo ${ }^{11}$ no posee problemas de multicolinealidad, ya que la media de los valores es igual a $1,44^{[12]}$, adicionalmente, es homocedastico, ya que el valor $\mathrm{Chi}^{2}$ es mayor $0,58^{[13]}$, por último, no posee problemas de normalidad, por lo que el modelo es confiable y permite concluir que las exportaciones al ser la única variable significativa dentro del modelo, aportan al crecimiento del sec- tor, por lo que si se requiere que el sector crezca un punto porcentual es necesario que las exportaciones aumenten a nivel nacional en un $620 \%$.

$\ln y=2,2201041+6,209745 \ln x+\xi[3]$

Dado lo anterior y bajo el hecho de que las exportaciones han disminuido al tiempo que las importaciones han

11 Este se modeló por Mínimos Cuadrados Ordinarios (MCO).

12 Cumple con la condición de ser menor a diez.

13 Cumple con ser mayor a 0,05 , por lo que se rechaza la hipótesis nula $\mathrm{H}_{0}$ : es heterocedástico. 
aumentado (figura 1), se hace imprescindible determinar una estrategia de internacionalización para el sector, que les permita aprovechar al máximo las oportunidades brindadas por el acuerdo comercial con la EU, para lo cual es importante establecer una herramienta que tenga en cuenta la RSE y de esta manera estimular el desarrollo sostenible alrededor del sector y, por ende, en el país, por tanto, en el primer paso se propone un modelo econométrico a partir del desarrollo de las primeras fases y los diferentes conocimientos de las investigadoras para determinar las significativas a la hora de construir la estrategia de internacionalización:

$x=f(r s e, c, p p s, t l c$, int, ino, $t i, a c, r a$, $c g, a)$

rse: Responsabilidad Social Empresarial, se medirá de acuerdo con una calificación promedio recibida en los programas de bienestar laboral, el tema ambiental y relacionamiento con la comunidad.

c: tasa de cambio real, se medirá a partir del índice de tasa de cambio real.

pps: políticas públicas sectoriales, se medirán a partir de la inversión realizada en el sector, desde la implementación de la política.

$t l c$ : tratados de libre comercio, se determinará a partir de la vinculación o no en uno de los tratados en los cuales se encuentra circunscrito el país.

int: innovación tecnológica, se medirá de acuerdo con la inversión realizada en nuevas tecnologías, ino: innovación organizacional, se medirá de acuerdo con las reestructuraciones organizacionales que realicen las empresas.

ti: tipo de internacionalización, en esta variable se determinará la percepción de los empresarios frente a diferentes tipos de internacionalización como franquicias, creación de clúster y sucursales internacionales, entre otras.

ac: acceso a crédito, se medirá de acuerdo con la valoración presentada por los empresarios.

ra: redes de apoyo, en esta variable se determina si la empresa accede a redes de apoyo empresariales, ya sean públicas o privadas.

cg: Competencia Global, esta variable presenta el sector confecciones en diferentes países.

a: Aranceles, se determinarán a partir de los que deban pagar en los diferentes países.

Adicionalmente, se tendrá en cuenta la percepción de los empresarios frente a cada una de estas variables con el fin de realizar el análisis cualitativo.

\section{CONCLUSIONES}

Se puede concluir que son muchos los retos del sector de confecciones, los cuales pueden ser abordados para efectos de la presente investigación desde dos ópticas distintas. Inicialmente, en materia comercial se marca la necesidad de potencializar las virtudes del sector, innovar en productos y procesos, garantizar productos de calidad a precios justos y abordar las necesidades del mercado que para este estudio se ve 
representado por la Unión Europea. Además, el sector presenta grandes desafíos en materia de Responsabilidad Social Empresarial, la cual debe tratar un aspecto tan concurrente en Colombia como es la informalidad laboral, esto sumado con los cuidados ambientales y las buenas prácticas empresariales configuran un escenario en el que debe primar el trabajo y compromiso del sector para garantizar el cumplimiento y la materialización de los objetivos propuestos.

Las anteriores perspectivas, a pesar de reflejar desde rutas diferentes dos contextos claves dentro del sector de confecciones en Colombia, evidencian también cierto grado de transversalidad en sus soluciones, puesto que al potencializar dichos aspectos, los efectos a mediano y largo plazo pueden impactar positivamente al sector, es decir, si las empresas comprometidas con las buenas prácticas empresariales lograran emitir estrategias para contrarrestar las problemáticas domésticas y llegaran a generar una imagen favorable dentro de los consumidores, esto, a su vez, influenciaría la decisión de compra y se obtendría de esta manera ganancias para el sector.

Lo mismo ocurriría en un escenario internacional, ya que los comportamientos socialmente responsables reflejan el compromiso con los objetivos de desarrollo sostenible, especialmente con el número ocho "Trabajo decente y crecimiento económico" (PNUD, 2018), desde el fomento de espacios laborales óptimos que lleven en sí al desarrollo de la persona, esto influenciaría sobre la percepción del sector y traería consigo resultados prósperos en temas de ventas, alianzas, patrocinios y respaldos.

Por tanto, el actual estudio desde la implantación de las fases de investigación y el diseño del modelo explicado previamente permitirá materializar en parte estas necesidades, a través de la construcción de una estrategia de internacionalización para el sector en mención, con un componente innovador que fortalezca y potencialice procesos al interior de este y, de igual manera, evidencie las ventajas de la implementación de estrategias de Responsabilidad Social Empresarial.

Lo anterior con miras a reunir esfuerzos que permitan la participación del sector de confecciones en un entorno de consumo globalizado, que le permitan aprovechar los beneficios del régimen especial de estímulo del desarrollo sostenible (SGP+) dentro del acuerdo comercial con la UE, incrementando sus exportaciones, lo que además pueda llevarlo a otros mercados internacionales viables y prósperos, aportando al desarrollo sostenible, junto con el acompañamiento del Estado colombiano, dado su compromiso en esta materia y el cual puede asumir desde diferentes ámbitos comerciales puntuales.

\section{REFERENCIAS BIBLIOGRÁFICAS}

CEDIS. (2009). La responsabilidad social empresarial. Una prioridad en el mundo empresarial moderno. Recuperado de https://www.cepal.org/ celade/noticias/paginas/8/12168/ respempresarialglobal.pdf 
Clavijo, P. y Ros. (2015). Para el debate científico, la ley de Thirlwall: una lectura crítica. Revista Investigación Económica, LXXIV(292), 11-40.

Recuperado de https://ac.els-cdn. com/S018516671500017X/1-s2.0S0185166

Comisión Europea. (2004). El Sistema de Preferencias Generalizadas de la Unión Europea (SPG). Recuperado de http://trade.ec.europa.eu/doclib/ docs/2004/march/tradoc_116449. pdf

Comisión Europea. (2018). Informe de la Comisión al Parlamento Europeo y al Consejo. Recuperado de https:// ec.europa.eu/transparency/regdoc/ rep/1/2018/ES/COM-2018-36-F1-ESMAIN-PART-1.PDF

Correa, J. G. Evolución histórica de los conceptos de evolución histórica de los conceptos de balance social. Semestre Económico, 87-102. Recuperado de http://www.scielo.org. co/pdf/seec/v10n20/v10n20a6.pdf

De la Vega, R. (2017). La innovación como estrategia competitiva para las empresas exportadoras. Analdex. Exponotas n. ${ }^{\circ}$ 50. Recuperado de http://www.analdex.org/wp-content/ uploads/1992/1

Delegación de la Unión Europea en Colombia y MINCIT. (2012). Acuerdo Comercial, Colombia Unión Europea. Delegación de la Unión Europea en Colombia. Recuperado de http://www.eeas.europa.eu/archives/ delegations/colombia/documents/ page_content/colombia_unio_n_europea_acuerdo_comercial_jul_11_ heavy_es.pdf

Echavarría, J. Condiciones laborales y productivas en microempresas de confección de la ciudad de Medellín. Revista CINTEX, 79-95.

Ekornes, K. (2013). Milton Friedman and Social Responsibility an Ethical Defense of Stockholder Theory. Recuperado de https://www.duo.uio. no/bitstream/handle/10852/38408/ Mertens_Filsosofi_Master. pdf?sequence $=$

Garrida, E. y Melé, D. (2004). Corporate Social responsibility Theories: Mapping the Territory. Journal of Business Ethics, 53(1). Recuperado de http://www.jstor.org/stable/ pdf/25123282.pdf

Hosseini, J. y Brenner, S. (1992). The Stakeholder Theory of the Firm: A Methodology to Generate Value Matrix Weights Business Ethics Quarterly. Recuperado de https:// www.jstor.org/stable/pdf/3857566. pdf?refreqid=excelsior\%3Aa5022f $0938 \mathrm{c} 74433$

Huanacuni, F. (2010). Buen vivir/vivir bien filosofía, políticas, estrategias y experiencias regionales andinas. Coordinadora Andina de Organizaciones Indígenas (CAOI).

Márquez,P. (2009). Crecimiento restringido por balanza de pagos en Colombia, Brasil y México. (Tesis inédita de doctorado). Universidad Nacional de Colombia. Recuperado de http:// www.fce.unal.edu.co/media/files/ documentos/Doctorado/Tes

Mincit. (2018). Tratados de libre comercio. Recuperado de http://www.tlc. gov.co/

Mincit. (2012). Acuerdo comercial Unión Europea-Colombia. Recuperado de https://eeas .europa.eu/sites/eeas/ files/04._acuerdo_comercial_cartilla.pdf 
Morales, E. (2016). Los negocios inclusivos en el sector textil en Colombia como estrategia de RSE (Responsabilidad Social Empresarial) para generar desarrollo local en Medellín. Bogotá: Universidad Militar Nueva Granada.

PNUD. (2018). Programa de Naciones Unidas para el Desarrollo. Recuperado de http://www.undp.org/content/ undp/es/home/sustainable-development-goals.html

Procolombia. (2017). Unión Europea un mercado de oportunidades para el sistema moda. Recuperado de http:// www.procolombia.co/publicaciones

Semana. (26 de agosto del 2018). El drama textil. Recuperado de https://www. semana.com/economia/articulo/cierre-temporal-de-fabricato/537670

Sen, A. (2000). El desarrollo como libertad. Gaceta Ecológica, Secretaría de Medio Ambiente y Recursos Naturales Distrito Federal, México, 14-20.

Thirlwall, A. (2011). The balance of payments constrained growth as an explanation of international growth rate differences. BPSL Quarterly Review, 64(259), 429-438. Recuperado de https://www.rspi.uniroma1. it/index.php/PSLQuarterlyReview/ article/viewFile/9 